INPLASY

PROTOCOL

To cite: Liu et al. An updated meta-analysis of whole-body vibration training to improve pain and function in patients with knee osteoarthritis.

Inplasy protocol 202130067. doi:

10.37766/inplasy2021.3.0067

Received: 18 March 2021

Published: 19 March 2021

Corresponding author:

Liu Liying

liuliying610610@163.com

Author Affiliation:

Shenyang Sport University, Qiansong East Road, Sujiatun

District, Shenyang City,

Liaoning Province,China

Support: Youth Seedling

Project.

Review Stage at time of this submission: Data extraction.

Conflicts of interest:

None declared.

\section{An updated meta-analysis of whole- body vibration training to improve pain and function in patients with knee osteoarthritis}

Liu, LY'; Sun, ML².
Review question / Objective: P: Patients with knee OA, I: The experimental group received whole body vibration training, The control group received rehabilitation treatment without WBVT. O:Main outcome indicators are WOMAC, VAS, NRS, 6MWT, BBS. S: RCT.

Condition being studied: Six databases including PubMed, Embase, Cochrane Library, CNKI, VIP, and Wanfang were searched up to January 2021. Randomized controlled trials(RCTs) of WBV for KOA were selected.

INPLASY registration number: This protocol was registered with the International Platform of Registered Systematic Review and Meta-Analysis Protocols (INPLASY) on 19 March 2021 and was last updated on 19 March 2021 (registration number INPLASY202130067).

\section{INTRODUCTION}

Review question / Objective: P: Patients with knee OA, I: The experimental group received whole body vibration training, The control group received rehabilitation treatment without WBVT. O:Main outcome indicators are WOMAC, VAS, NRS, 6MWT, BBS. S: RCT.

Condition being studied: Six databases including PubMed, Embase, Cochrane Library, CNKI, VIP, and Wanfang were searched up to January 2021. Randomized 
controlled trials(RCTs) of WBV for KOA were selected.

\section{METHODS}

Participant or population: Patients with knee OA, 601 participants.

Intervention: Whole body vibration training.

Comparator: The control group received rehabilitation treatment without WBVT.

Study designs to be included: Randomized controlled trials(RCTs) of WBV for KOA were selected. After screening, data extraction, quality evaluation and risk assessment, meta-analysis was carried out with RevMan 5.4.1 software. 13 RCT trials with 601 participants provided data for the meta-analysis.

Eligibility criteria: KOA patients without injury and surgery.

Information sources: A total of 13 documents were obtained after retrieval, including 9 documents in English and 4 documents in Chinese; 192 duplicate documents were excluded through preliminary screening, 549 irrelevant documents were excluded through reading titles and abstracts, and documents that did not meet the inclusion criteria were eliminated after further reading of the full text. 130 articles, 43 articles were included qualitatively, and 13 articles were finally included quantitatively. The total sample size was 601 cases, the sample size of the experimental group was 323 cases, and the sample size of the control group was 278 cases.

Main outcome(s): Meta-analysis results showed that WBVT could reduce VAS and NRS pain score and WOMAC pain score and WOMAC function score, Improve Lysholm score, the effect is obvious and are statistically significant.

Additional outcome(s): The significant differences were shown in BBS, No significant difference was shown in TUGT and 6MWT. Conclusion WBVT can relief the pain and improve function to a certain extent. But still need to expand the number of participants for Clinical medicine.

Quality assessment / Risk of bias analysis: All of the 13 included articles mentioned "random" or "randomized controlled trials", 5 mentioned the use of random number tables or computer-generated sequences and other specific random methods, and 7 papers mentioned specific allocation concealment measures, 1 One article was blinded by the experimenter and the subject, and seven articles were blinded to evaluate the outcome indicators. In addition, 6 articles reported on the treatment of loss to follow-up or missing data, and 13 articles were not sure whether there were other biases.

Strategy of data synthesis: REVMAN5.4.1.

Subgroup analysis: None.

Sensitivity analysis: None.

Country(ies) involved: China.

Keywords: Knee osteorthritis; Whole body vibration training; meta-analysis; joint function; pain.

Contributions of each author:

Author 1 - Liu Liying.

Author 2 - Sun Mingli. 